\title{
NUTATION AND REFERENCE FRAME
}

\author{
N. CAPITAINE \\ Observatoire de Paris, URA1125 \\ 61, avenue de l'Observatoire \\ 75014 Paris, France
}

\section{Introduction}

The reference frames are of fundamental importance in all kinds of the precession and nutation studies involving the theory, the coordinate transformation and the observations. The aim of this paper is to review all the frames used in such studies and to lead to a better consistency between them in order that theory and reductions of observations be referred, as close as possible, to the frames to which observables are actually sensitive.

\section{The reference frames for the theory}

The equations of Earth rotation can be expressed either as Euler equations in the Terrestrial Reference System (TRS), or as perturbation theory in the Celestial Reference System (CRS) (Kinoshita 1977). Euler equations are transformed to the CRS in the astronomical approach (Woolard 1953) and solved by the method of variation of the parameters, whereas, in the geophysical approach (Melchior 1971), the solutions, first obtained in the TRS, are transformed to the CRS and then solved by an integration with respect to time.

The two approaches are theoretically equivalent, but the astronomical approach provides a better precision in the CRS than the geophysical one due to the small divisors appearing in the integration w.r.t. time. In the mixed astronomical/geophysical approach, as in the IAU 1980 nutation theory, the solutions are obtained in the CRS by convolution from rigid Earth nutation in the CRS and non rigid Earth generalized solutions in the TRS (Wahr 1981). 
The theory of Earth rotation around its center of mass provides a geocentric reference frame moving with precession and nutation through the expressions :

- of precession and nutation of an axis which can be the axis of angular momentum, the instantaneous axis of rotation or the Tisserand mean axis, the differences between them being given by the Oppolzer terms in the CRS or diurnal nutation in the TRS.

- of the angle of Earth rotation along the equator from an arbitrary origin which is classically the true equinox, but can also be the uniform equinox, without nutation, as proposed by Atkinson and Sadler (1951) or the non-rotating origin (NRO) as proposed by Guinot (1979).

The theory of nutation generally gives (Kinoshita 1977, Kinoshita and Souchay 1990) the nutation angles referred to the ecliptic of date (Seidelmann 1982), except Woolard (1953) who has developed the theory referred to the ecliptic of epoch. On the contrary, numerical integrations always provide the nutation angles referred to the fixed ecliptic.

\section{The transformation between CRS and TRS}

The general form of the coordinate transformation from the CRS to the TRS at the date $t$ is the product of three matrix transformations, one for precession and nutation, one for the rotation of the Earth and one for polar motion.

If the coordinate transformation refers to the equinox :

- the angle for Earth rotation is Greenwich True Sidereal Time, GST, which includes both Earth rotation and accumulated precession and nutation,

- the coordinate transformation for precession and nutation is classically the product of six rotations, first for the precession angles and then for the nutation angles referred to the ecliptic of date.

If the coordinate transformation is referred to the NRO :

- the angle for Earth rotation is the stellar angle, $\theta$, reckoned from the NRO and including therefore only the specific Earth's angle of rotation,

- the transformation matrix for precession and nutation is the product of only four rotations of which parameters are the coordinates of the Celestial Ephemeris Pole (CEP) in the CRS and the accumulated rotation, $s$, of the NRO on the equator due to celestial motion of the CEP (Capitaine 1990).

A change in the model for precession and nutation will give a change in GST of the same order of magnitude, but a change in $\theta$ of the second order and the reference to the kinematically defined NRO leads to an exact definition of UT1 (Capitaine and Gontier 1993). 


\section{Reference frames and observations}

The Earth rotation is classically observed through the measured Earth Orientation Parameters (EOP), as variations of the universal time UT1-UTC and changes in the terrestrial orientation of the axis of the celestial pole. The celestial pole offsets can also be provided by VLBI or LLR observations as well as by long series of optical astrometry .

The estimated EOP are referred to the equator of the CEP to which the observations are actually sensitive, but not to the ecliptic, even in the case of the LLR observations. The celestial pole offsets are corrections to the coordinates of the CEP in the CRS.

The relationship between theory and observations is strongly dependent on the intermediate reference frame which is used in the transformation between CRS and TRS, on the method used for the reduction of the observations and on the spatial and temporal resolution of the measured EOP (Brzezinski and Capitaine 1993). For example, the sub-daily determinations make less clear the distinction between high-frequency polar motion, which affects the terrestrial coordinates of the CEP and nutation which affects its celestial coordinates.

\section{Concluding remarks}

In order to take all the scientific benefit of the present observational accuracy, it is necessary that the frames considered in nutation studies be defined to be as close as possible to those to which observables are sensitive. A first improvement has been given in the IAU 1980 theory of nutation by the adoption of the CEP, which is really observable, instead of the pole of rotation, which is not directly observable by the present techniques.

The techniques presently used for deriving precession and nutation being sensitive to the rotation of the Earth and to the motion of the equator in space as well, but not to the motion of the ecliptic, it would be better that:

- theory gives the nutation angles with respect to the fixed ecliptic,

- astronomical standards give independently the motion of the equator and the motion of the ecliptic and not a mixed constant of precession which includes both motions,

- the coordinate transformation from the CRS to the TRS clearly separates Earth rotation from precession and nutation.

\section{References}

Atkinson, R.d'E and Sadler, D.H. (1951), Mon. Not. R. Astr. Soc., Vol. 111, pp. 619. Brzezinski, A. and Capitaine, N. (1993), J. Geophy. Res., Vol. 98, B4, pp. 6667. Capitaine, N. (1990), Celest. Mech. Dyn. Astr., Vol. 48, pp. 127. 
Capitaine, N. and Gontier, A.-M. (1993), Astron. Astrophys., Vol. 275, pp. 645.

Guinot, B. (1979), in: Time and the earth rotation, eds D D. McCarthy and J.D. Pilkington, IAU Symposium 82, pp. 7.

Kinoshita, H. (1977), Celest. Mech., Vol. 15, pp. 277.

Kinoshita, H. and Souchay, J. (1990), Celest. Mech., Vol. 48, pp. 187.

Melchior, P. (1971), Celest. Mech., Vol. 4, pp. 190.

Seidelmann (1982), Celest. Mech., Vol. 27, pp. 79.

Wahr J.M. (1981), Geophys. J. R. astron. Soc., Vol. 64, pp. 651.

Woolard (1953), Astr. Pap. Amer. Eph. Naut. Almanac, Vol. XV(I), pp. 1. 